\title{
Synthesis, Characterization, and Thermal Property of Functional Biodegradable Polycaprolactone
}

\author{
Wei Na Gao ${ }^{1, a}$, Xiong Yan Zhao ${ }^{1,2, b^{*}}$ \\ ${ }^{1}$ College of Material Science and Engineering, Hebei University of Science and Technology, \\ Shijiazhuang, 050018, P. R. China \\ ${ }^{2}$ State Key Laboratory Breeding Base___Hebei Province Key Laboratory of Molecular Chemistry \\ for Drug, Shijiazhuang 050018, China \\ aemail: gaogaoweina@126.com, ${ }^{b^{*}}$ email: zhaoxy66@126.com
}

Keywords: polycaprolactone, functional groups, thermal property, polyesters

\begin{abstract}
A new approach for preparing polycaprolactone (PCL) with functional groups was introduced, by which three kinds of functional group-terminated PCLs (PCL-OH, PCL-Boc and PCL- $\mathrm{NH}_{2}$ ) were designed and synthesized. The composition, structure, and thermal property of these functional PCLs were characterized by FTIR, NMR and TGA. The results show that PCL-OH, PCL-Boc and PCL-NH $\mathrm{N}_{2}$ were synthesized successfully. This new versatile synthetic platform can provide a large family of biodegradable, functional group-terminated copolyesters which are useful for the preparation of novel biodegradable polyester elastomer.
\end{abstract}

\section{Introduction}

In order to minimize the long-term effect of biomaterials on human body, the medical biomaterials which are implanted in the body are only expected to play a temporary role, and will gradually degrade or assimilate with the regeneration of tissues or organs. That is to say, medical biomaterials should not only have good biocompatibility, but more importantly, they should also be biodegradable. Biodegradable polyester is a kind of polyester which can degrade into low-molecular compounds under natural conditions[1]. Since the 1980s, biodegradable polyester was investigated rapidly after the US Food and Drug Administration approved the use of natural or biodegradable materials used as tissue engineering materials. Polycaprolactone (PCL) has excellent biodegradability and shape memory characteristics[2], and widely used in surgical sutures, tissue repair and wound dressings[3-6]. Since the biocompatibility and hydrophilic of PCL are poor, Thus, development of novel biodegradable functional polyesters is now becoming one of the most important and challenging issues in the biomedical field [7-8].

The key in preparing biodegradable elastomers based on chemical crosslinking is the development of novel biodegradable multifunctional-group-terminated copolyesters, which can be used as multifunctional macromonomer for the synthesis of network biodegradable elastomers. In this paper, a new approach for preparing polycaprolactone (PCL) with functional groups was introduced, by which three kinds of functional group-terminated PCLs (PCL-OH, PCL-Boc and PCL-NH $\mathrm{N}_{2}$ ) were designed and synthesized. (see Scheme 1). One of the objectives of the work was to develop novel biodegradable multifunctional group-terminated copolyester PCL which can be used as multifunctional macromonomer for the synthesis of novel network biodegradable polyester elastomers. 


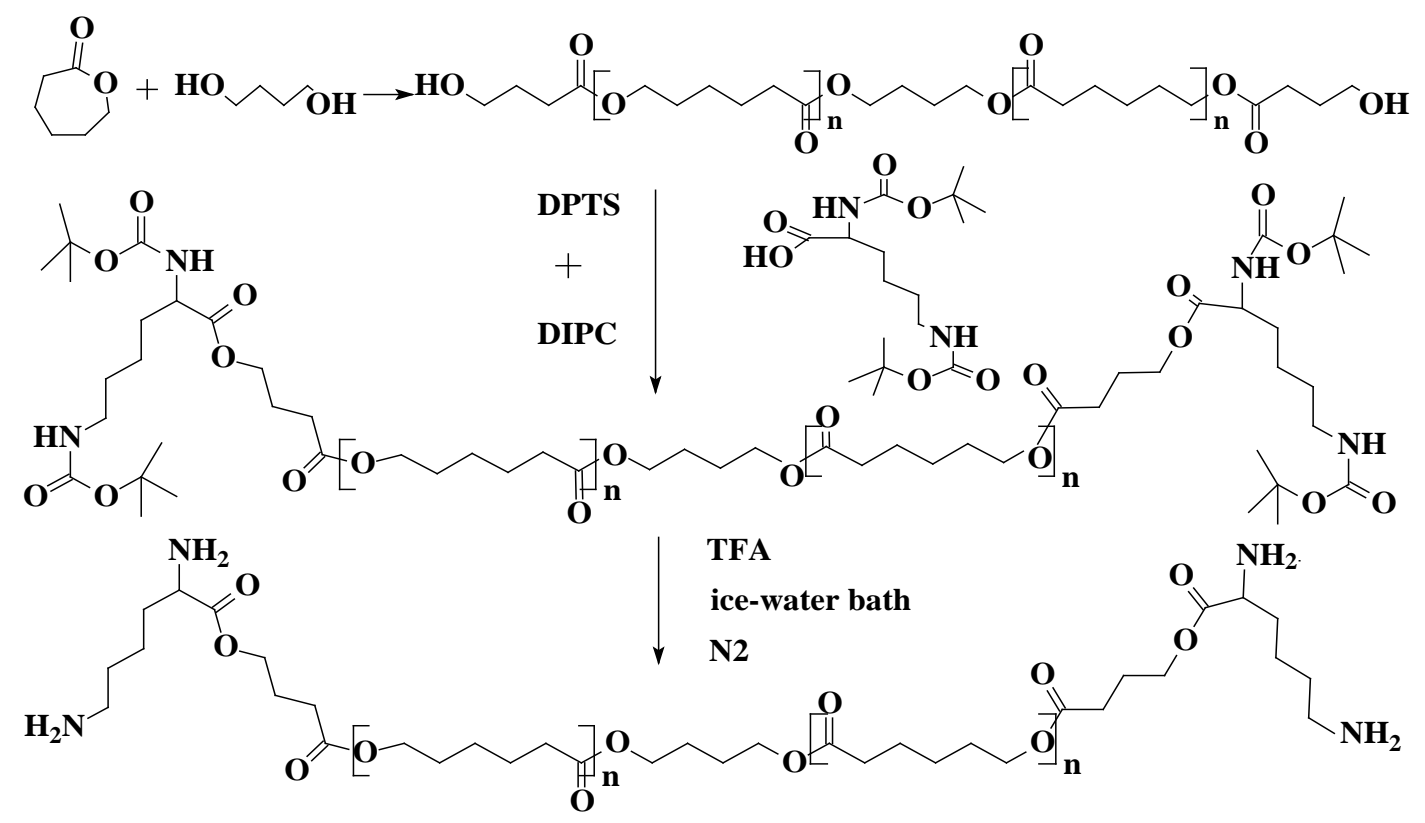

Scheme 1 The synthetic route of functional group-terminated PCLs.

\section{Experimental}

Materials.1,3-diisopropyl-carbodiimide (DIPC), Stannous octoate and 1,4-butanediol were purchased from Aldrich, caprolactone were purchased from Jinan Dai Gang biotechnology company. N1, N2- bis (tert- butoxycarbonyl)-L-lysine was extracted from N1, N2-bis(tert-butoxycarbony)-L-lysine dicyclohexylamine, Tetrahydrofuran were purchased from Tianjin Fuyu Fine Chemicals Co. Ltd.

Synthesis of PCL-OH. Caprolactone $(0.04 \mathrm{~mol}), 0.2880 \mathrm{~g}$ of 1,4 -butanediol and $0.0912 \mathrm{~g}$ of stannous octoate were added to a three-neck flask equipped with a condenser. Under N2 atmosphere, the system was then heated to $140 \mathrm{oC}$ in an oil bath with magnetic stirring. The reaction was continued for $4 \mathrm{~h}$. The system was cooled to $60 \mathrm{C}$ after completion of the reaction. A mount of THF was pured into the three-neck flask, the mixture was stirred until the product was dissolved in THF. The filtrate was obtained with vacuum extraction filtering, and then poured the filtrate into hexane of fourfold volume. The mixture was stirred for a while, and then kept undisturbed overnight. The white precipitate was obtained and dried in the vacuum oven.

Synthesis of PCL-Boc. 3g PCL-OH, 0.3g N1, N2- bis(tert- butoxycarbonyl)-L-lysine, 0.084g DPTS and 0.1092g DIPC were dissolved in $30 \mathrm{ml}$ dichloromethane in a three-neck flask. The solution was then heated to $25 \mathrm{oC}$ in an oil bath with magnetic stirring. This reaction continued for $24 \mathrm{~h}$ under N2 atmosphere. After completion of the reaction, the filtrate was obtained with vacuum extraction filtering, and then poured the filtrate into ice-cold methanol of fourfold volume. The mixture was stirred for a while, and then kept undisturbed overnight. The white precipitate was obtained and dried in the vacuum oven.

Synthesis of PCL-NH2. 1.5g of PCL-Boc was dissolved in $30 \mathrm{ml}$ dichloromethane in a three-neck flask. Then, it was sealed under N2 atmosphere with an ice bath, and then $4.5 \mathrm{ml}$ trifluoroacetic acid was added into the mixture. The reaction kept for $4 \mathrm{~h}$. The filtrate was obtained with vacuum extraction filtering, and then poured the filtrate into hexane of fourfold volume. The mixture was stirred for a while, and then kept undisturbed overnight. The white precipitate was obtained and dried in the vacuum oven.

Characterizations. ${ }^{1} \mathrm{H}-\mathrm{NMR}$ spectra were recorded on Bruker 400 NMR spectrometer at 400 $\mathrm{MHz}$ in deuterated chloroform with tetramethylsilane (TMS) as an internal standard. Fourier transform infrared spectroscopy was measured by Fourier Transform Infrared Spectrometer (Model FTS-13). Thermal stability measurements were carried out on a thermogravimetric analysis 
(TGA)/DSC 1/1100 HT (Mettler Toledo) thermogravimetric analyser under a nitrogen flow at a heating rate of $10 \mathrm{C} \mathrm{min}^{-1}$.

\section{Result and Discussion}

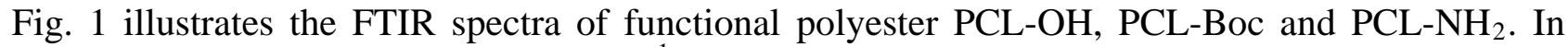
curve(a)the absorption band at $3450 \mathrm{~cm}^{-1}$ represents the hydroxyl groups stretching vibration of PCL-OH component, which indicated the existence of -OH end-groups in the copolyester PCLA-OH. In curve(b). The absorption peak of $3450 \mathrm{~cm}^{-1}$ disappeared, and the secondary amine $\mathrm{N}-\mathrm{H}$ deformation vibration appeared at $1500 \mathrm{~cm}^{-1}$, which indicated that PCL-Boc was synthesized. In curve(c), the secondary amine $\mathrm{N}-\mathrm{H}$ deformation vibration disappeared, and the primary amine $\mathrm{N}-\mathrm{H}$ bending absorption peak appeared at $1650 \mathrm{~cm}^{-1}$, which indicated that Boc protecting group was removed, PCL- $\mathrm{NH}_{2}$ was synthesized.

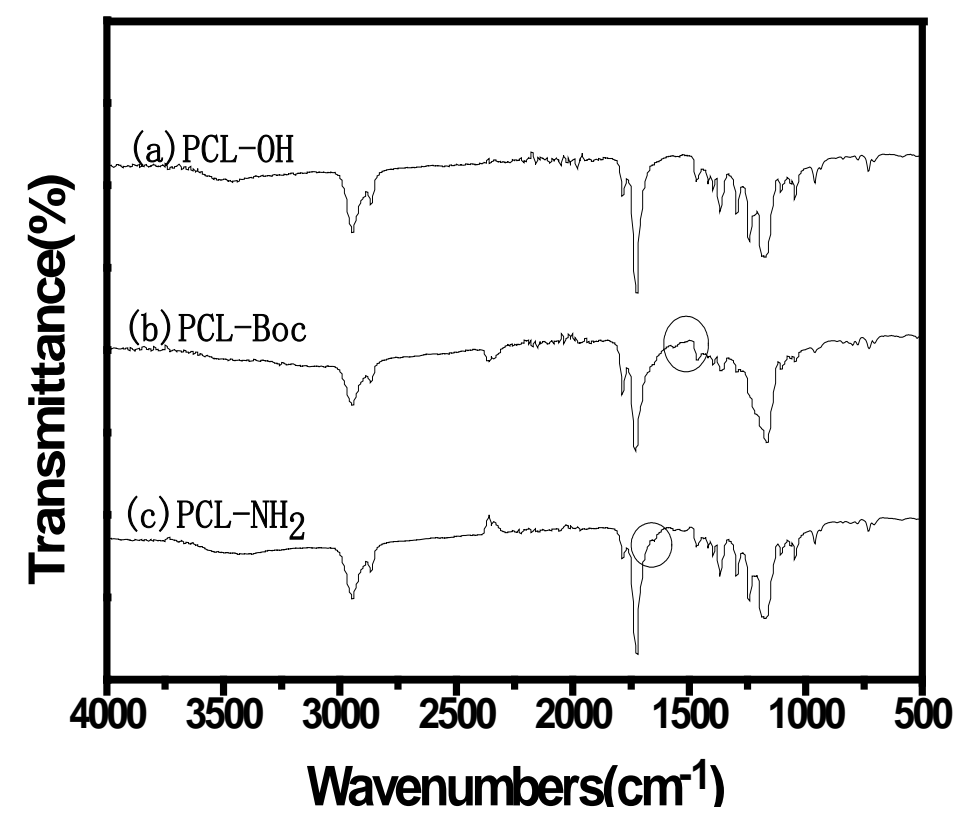

Fig. 1. FTIR spectra of (a) PCL-OH, (b) PCL-Boc, and (c) PCL-NH 2

The TGA curves of PCL-OH, PCL-Boc and PCL-NH $\mathrm{NH}_{2}$ were shown in Fig. 2. It was shown that the PCL-OH had superior thermal properties with the initial decomposition temperature of 258C, while the PCL-Boc decomposed at $253 \mathrm{C}$ and the PCL- $\mathrm{NH}_{2}$ decomposed at $252 \mathrm{C}$, which were lower than that of PCL-OH. Furthermore, the degradation temperature of $50 \%$ weight loss was 340C and 338C for PCL-OH and PCL-Boc, respevtively, obviously higher than that of PCL-NH $\mathrm{N}_{2}$ (328C). The low thermal stability for functional polyester PCL- $\mathrm{NH}_{2}$ may be due to the higher activity of the amino groups. 


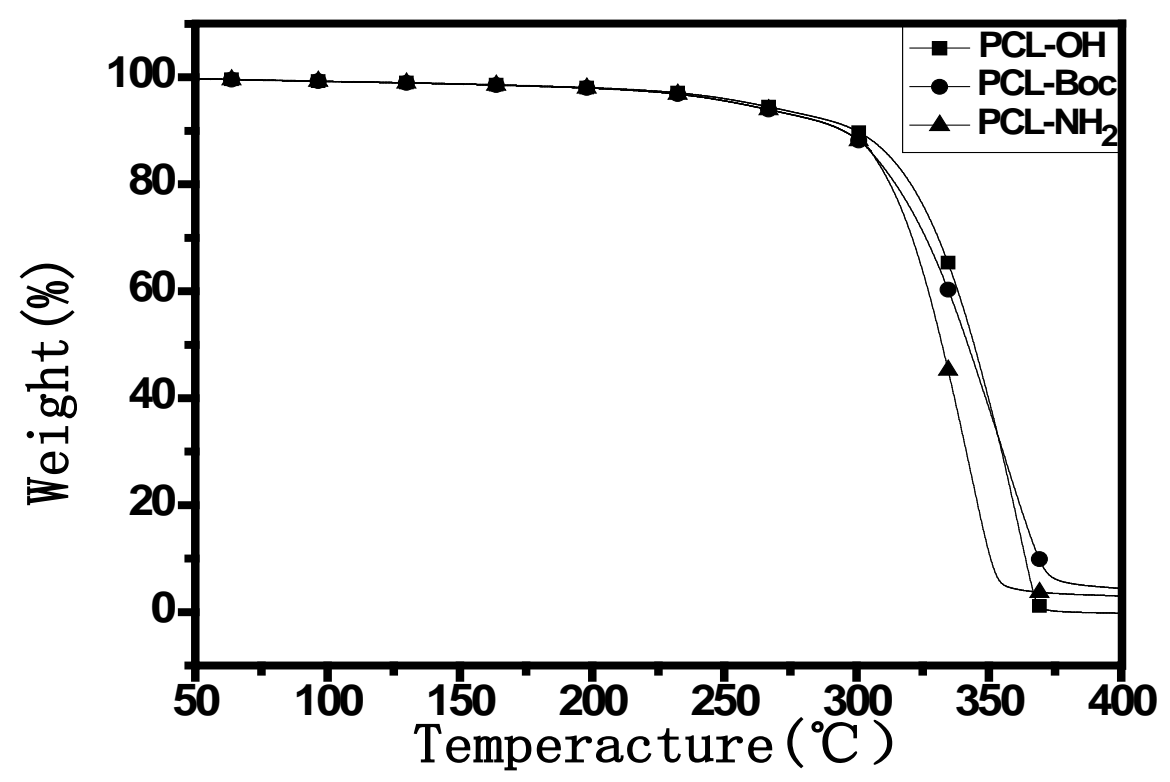

Fig. 2. TGA curve of PCL-OH, PCL-Boc, PCL-NH 2

\section{Conclusions}

In this article, a new method for the synthesis of biodegradable multifunctional-group-terminated copolyesters is explored, by which PCL-OH, PCL-Boc and PCL-NH2 were designed and synthesized. Amino-functional polyester has the lowest thermal degradation temperature, which may be due to the higher activity of the amino groups. The obtained functional polyesters might have huge potential applications in biomedical fields, such as drug delivery and release, tissue engineering, and modern medical equipment and instruments.

\section{Acknowledgements}

The Project was supported by the National Special Found for Scientific Research in the Public Interest of China (Grant No. 201310151).

\section{References}

[1] B.F. Deng, C.S. Huang, Z.J.Ye, R.T. Ren and Z.X.Huang: Materials Development and Application Vol. 27 (2012), p. 72

[2] C.Q. Xie, Y.L. Zhou and J.J.Ma: Science and Technology of Plastics Vol. 37 (2009), p. 100

[3] H.Z.Mai, Z.Y.Wang, B.Yan and Y.M. Zhao: Chemical Research and Application Vol. 14 (2002), p. 534

[4] C. Vaida, P. Meia and K. Kunna: Macromolecular Bioscience Vol. 10 (2010), p. 925

[5] Q. Tu, J.C. Wang, R. Liu, Y. Chen, Y. Zhang, D.-E. Wang, M.-S. Yuan, J. Xu and J. Wang: Colloids and Surfaces B: Biointerfaces Vol. 108 (2013), p. 34

[6] S. Theiler, S. E. Dlamantouros and S. Jockenhoevel: Polymer Chemistry, Vol. 2 (2011), p. 2273

[7] S. V. Bael, T. Desmet and Y. C. Chai: Materials Science and Engineering C, Vol. 33 (2013), p. 3404

[8] M. Domingos, F. Intranuovo and A. Gloria: Acta Biomaterialia, Vol. 9 (2013), p. 5997 